\title{
CONTINUOUS APPROXIMATIONS FOR LONG-TERM NUMERICAL SIMULATIONS OF THE SOLAR SYSTEM
}

\author{
S. Rehman (1) \\ Department of Mathematics, \\ University of Engineering and Technology, Lahore, Pakistan, \\ srehman@uet.edu.pk \\ (Received 16 January 2021; in final form 28 July 2021; accepted 26 August 2021; published online 30 September 2021)
}

\begin{abstract}
We present and analyse the performance of different combinations of four higher-order numerical integrators and up to nine interpolation schemes applied to the problem involving the Sun and four Gas-giants (outer planets), namely, Jupiter, Saturn, Uranus, and Neptune. The Hermite interpolation schemes obtained by one, two, and three time-step and interpolants for ODEX2 and ERKN integrators are considered in this paper. The interpolants are a special example of an interpolation scheme, which produce an approximation that is continuous across one step and across the complete interval of integration. The interpolants are quite expensive in comparison with the other interpolation schemes. Therefore, one of the objectives of this paper is to investigate the possibilities of replacing the interpolants of certain integrators by other interpolation schemes, perhaps at a cost of a little bit of accuracy. The experiments are performed to examine the error growth in the positions, velocities, and relative error in energy and angular momentum using different combinations of integrators and interpolation schemes over a long interval of integration, as long as 100 million years for the Jovian problem with local error tolerances ranging from $10^{-16}$ to $10^{-08}$.
\end{abstract}

Key words: Jovian problem, interpolation schemes, long-term simulations.

DOI: https://doi.org/10.30970/jps.25.3901

\section{OVERVIEW}

The interpolation schemes play a key role in $N$ body simulations, in-particular, when detecting closeencounters between a massive body and a test particle in the Solar System dynamics. It is possible that a closeencounter occurs within the time-step and not at the end points. Such a close-encounter would not be detected if we only have numerical approximations at the end points. One way of detecting such close-encounters is to generate numerical approximations within the integration time-step by using a far smaller time-step when the test particle is near the massive body compared with when it is far from the massive body. In practice, this approach would be inefficient because all bodies, including those that are not undergoing close-encounters, would be integrated with the smaller time-step, resulting in a possibly tremendous increase in CPU-time. Thus, an alternative approach is needed to avoid this inefficiency. The numerical approximation at the mesh points may be extended to a continuous approximation, which provides the numerical solution at any point $t$, for $t$ within the time-step interval. Large numbers of numerical integrators and associated interpolation schemes for performing $N$-body simulations have been developed and implemented; see, for example, [9, 18-22].

In the next section, we illustrate the physical formulation of the Jovian problem, integrators and interpolation schemes applied to the Jovian problem, different types of errors, estimation of the maximum global error by sampling the norm with different sets of data points. In Section III, we present continuous approximations of variable-step-size integrators, and a schematic that presents the classification of the errors as a local segment of the numerical solution. Numerical testing is done in Section IV involving comparisons of combinations of different integrators and interpolation schemes, and overall summary is presented in Section V. All computer programs are written in FORTRAN and we have used Matlab to analyse the results. The graphs are smoothed with the Matlab filter command.

\section{BACKGROUND}

The Jovian problem models the orbital motion of the Sun and four Gas giants (outer planets), namely, Jupiter, Saturn, Uranus, and Neptune, interacting with one another through Newtonian forces [23]. The shortest orbital period for the Jovian problem is 4331 days (Jupiter). The Jovian planets collectively drive much of the dynamics of our Solar System. Therefore, the Jovian problem is frequently used in numerical approximations, inparticular, when simulations are performed over a long interval of integration. These longterm numerical simulations provided more insight into the Solar System dynamics, which went further than that given by analytic theories. Let $r_{i}=\left[x_{i}, y_{i}, z_{i}\right]^{T}, i=1, \ldots, 5$, denote the position of the $i^{\text {th }}$ body of the Jovian problem in a threedimensional Cartesian coordinate system with the origin at the barycentre of the bodies. The equations of motion for the $i^{\text {th }}$ body of the Jovian problem can be written as

$$
r_{i}^{\prime \prime}(t)=\sum_{j=1, j \neq i}^{5} \frac{\mu_{j}\left(r_{j}(t)-r_{i}(t)\right)}{\left\|r_{j}(t)-r_{i}(t)\right\|_{2}^{3}}, \quad i=1, \ldots, 5,
$$

where $\|.\|_{2}$ is the $L_{2}$-norm, and $\mu_{j}$ denotes the gravitational constant $G$ times the mass $m_{j}$ of the $j^{\text {th }}$ body, i.e., $\mu_{j}=G m_{j}$, distance is expressed in astronomical units, time in Earth days, whereas the mass $m_{j}$ in Solar mass. 


\section{S. REHMAN}

For each body of the Jovian problem we have a $2^{\text {nd }}$-order differential equation for the $x^{-}, y^{-}$, and $z$-components, giving us $152^{\text {nd }}$-order differential equations in total. For efficiency purposes, we use the symmetry of interactions for evaluating the acceleration for the Jovian problem. If we consider the individual terms in the summation, then it can be observed that

$$
\frac{\left(r_{j}(t)-r_{i}(t)\right)}{\left\|r_{j}(t)-r_{i}(t)\right\|_{2}^{3}}=\frac{-\left(r_{i}(t)-r_{j}(t)\right)}{\left\|r_{j}(t)-r_{i}(t)\right\|_{2}^{3}} .
$$

Once this term for $r_{j}$ is obtained, we can easily update the acceleration for the $2^{\text {nd }}$ body by using symmetry. It has been observed that using symmetry, the subroutine for evaluating the force term reduces to approximately half of the CPU-time.

Explicit Runge-Kutta-Nyström (ERKN) methods for the numerical approximation of $2^{\text {nd }}$-order differential equations were proposed by E. J. Nyström in 1925 [12]. ERKN methods reduce the computational cost considerably, compared to explicit Runge-Kutta (ERK) methods applied to the corresponding equivalent system of first-order differential equations. For example, an order-five ERKN method requires only four function evaluations per integration time-step, whereas an ERK method of the same order requires at least six function evaluations [10]. The efficiency of an ERKN method depends on the technique for controlling the error in the numerical solutions. An adaptive step-size technique is one of the possible options of controlling the error that permit control of the estimated local error. A pair of different orders formulae is implemented in such a manner that the function evaluations of both methods are identical. Usually, the numerical approximation is performed by a higher-order method and the error is obtained by the lower-order method to gain maximum efficiency. Here, we use two ERKN integrators: a 9-stage ERKN689, 68 FSAL pair and a 17-stage ERKN101217, 10-12 nonFSAL pair [2].

Extrapolation provides a strong means of accelerating the convergence of solutions that arise from discretization methods, and also have strong connections with, for example, projection methods, continued functions and Padé approximations. For the direct numerical approximations of $2^{\text {nd }}$-order systems of differential equations, Hairer et al. [10] developed an extrapolation code $O D E X 2$. This code is based on the explicit midpoint rule along with order selection and a step size control technique. The extrapolation code $O D E X 2$ is efficient for all tolerances, in-particular for high precision.

Störmer's methods are an important class of numerical methods for the numerical approximation of systems of $2^{\text {nd }}$-order differential equations [14]. Störmer's methods have long been implemented for long-term numerical simulations of the Solar System dynamics [9]. Grazier [9] suggested the $13^{\text {th }}$-order, fixed step-size Störmer method that uses backward differences in summed form, summing from the highest to the lowest differences. For the Jovian problem, the numerical testing in [8] depicts that the error in energy and the phase error grow as $t^{1 / 2}$ and $t^{3 / 2}$, respectively, to within numerical uncertainty when the step size is approximately 4 days of Jupiter's orbital period. This particular selection of the step-size guarantees that the local truncation error is well below machine precision. In this work, we use the order-13, fixed step-size Störmer method and associate to it as $\bar{S}-13$ integrator.

For the continuous approximation of the Jovian problem, the interpolation schemes used in this paper are: one-step (cubic and quintic Hermite interpolation schemes), two-step, and three-step Hermite interpolation schemes. The cubic Hermite interpolation polynomial is of degree 3, whereas the quintic, two-step, and threestep are of degrees 5, 8, and 11, respectively. For one time-step, the cubic Hermite interpolation polynomial interpolates the data $\left(t_{n-i}, y_{n-i}\right)$ and $\left(t_{n-i}, y_{n-i}^{\prime}\right)$ at time $t_{n-i}$, for $i=1$ and 0 and can be expressed as

$$
\begin{aligned}
P_{3}(t) & =(\tau-1)^{2}(2 \tau+1) y_{n-1}+(\tau-1)^{2} \tau H y_{n-1}^{\prime} \\
& +\tau^{2}(3-2 \tau) y_{n}+\tau^{2}(\tau-1) H y_{n}^{\prime}
\end{aligned}
$$

where, $H=t_{n}-t_{n-1}$ and $\tau=\left(t-t_{n-1}\right) / H$. Since the values of $y$ and $y^{\prime}$ are interpolated at both ends of each time-step, the piecewise defined approximation obtained from the cubic polynomial is continuous and has a continuous first derivative.

The quintic polynomial also interpolates the data involving position, velocity, and acceleration at time $t_{n-i}, i=1,0$. The values of $y, y^{\prime}$, and $y^{\prime \prime}$ are interpolated at both ends of each time-step. Therefore, the piecewise defined approximation obtained from the quintic polynomial is continuous and has continuous first- and second-derivatives. Similarly, the two-step polynomial interpolates the data involving position, velocity, and acceleration at time $t_{n-i}, i=2,1,0$; whereas, the three-step interpolation polynomial interpolates the data involving position, velocity, and acceleration at time $t_{n-i}, i=3,2,1,0$. For comprehensive details of the onestep, two-step, and three-step interpolation schemes, we refer to [17]. Solution interpolants for ODEX2 integrator and ERKN integrators are discussed in Sections III A and IIIB, respectively.

Now, we define different types of errors used in this paper. We use the notation $y_{\text {true }}(t)$ to denote the true solution and $y_{\text {num }}(t)$ to denote the approximate solution. The difference is monitored time-wise by considering the global error in $y$ as

$$
y_{\text {num }}(t)-y_{\text {true }}(t) .
$$

The norm of the global error in $y$ at time $t$ is then

$$
\left\|y_{\text {num }}(t)-y_{\text {true }}(t)\right\|_{2} \text {. }
$$

The calculation of the global error is discussed later. A large number of integrators, for example, Runge-KuttaNyström [12], and Störmer [14] can be used to find $y_{\text {num }}(t)$ for $t \geq 0$. This leads to numerical solutions $y_{n}=y_{\text {num }}\left(t_{n}\right)$ and $y_{n}^{\prime}=y_{\text {num }}^{\prime}\left(t_{n}\right)$ at times $t_{n}=t_{0}+n h$, $n=1,2, \ldots$, where $h$ can depend on $n$. The approximation over the continuous time interval can then be computed using (local) interpolation. 
From one time-step to the next, the local problem is solved, which is defined as

$$
\begin{aligned}
& u_{n}^{\prime \prime}=f\left(t, u_{n}\right), \quad u_{n}\left(t_{n-1}\right)=y_{n-1}, \\
& u_{n}^{\prime}\left(t_{n-1}\right)=y_{n-1}^{\prime}, \quad t \in\left[t_{n-1}, t_{n}\right],
\end{aligned}
$$

where $u_{n}(t)$ is the true local solution on the $n^{\text {th }}$ interval. The initial conditions $y_{n-1}$ and $y_{n-1}^{\prime}$ are the values of the approximate solution at the end of the $(n-1)^{\text {st }}$ step. Hence, for the local problem, the DEs are the same as for the original problem, while the initial conditions depend upon the numerically approximated solution. Because, in general, the approximation $y_{n-1}$ is not equal to the true solution $y_{\text {true }}\left(t_{n-1}\right)$, there is a difference between the error obtained in this local step and the global error. The norm of the local error is defined as

$$
\left\|y_{\text {num }}(t)-u_{n}(t)\right\|_{2}
$$

where $t \in\left[t_{n-1}, t_{n}\right]$. In general, the global error cannot be calculated because the true solution is not known.

The estimated global error in the position over each timestep, $n$, is defined in terms of an accurate numerical solution (computed in quadruple precision), $y_{\text {ref }}(t)$, and an underlying polynomial approximation is introduced at each step, $P_{n}(t)$. We then estimate global error for $t \in\left[t_{n-1}, t_{n}\right]$ by evaluating $P_{n}(t)-y_{\text {ref }}(t)$ at $k$ evenly spaced sample points over the step. A more detailed description of $y_{\mathrm{ref}}(t), k, P_{n}(t)$, and the definition of the estimated global error in the position is presented later in this section.

The global error in the position for all $t \in\left[t_{0}, t_{f}\right]$, where $t_{f}$ is the pre-specified final value of $t$ in the integration, is more difficult to define and estimate, because we need a continuous approximation to $y(t)$, and not just the discrete values $y_{1}, y_{2}, \ldots$. We obtain the continuous approximation by first forming a polynomial $P_{n}(t)$ that approximates the local solution on the interval $\left[t_{n-1}, t_{n}\right]$. The numerical approximation is then the piecewise-defined function

$$
y_{\text {num }}(t)= \begin{cases}P_{1}(t), & t_{0} \leq t \leq t_{1} \\ P_{2}(t), & t_{1} \leq t \leq t_{2} \\ \vdots & \\ P_{n}(t), & t_{n-1} \leq t \leq t_{n} \\ \vdots & \end{cases}
$$

where we have assumed $P_{n-1}\left(t_{n-1}\right)=P_{n}\left(t_{n-1}\right)$, an assumption that holds throughout the paper. The corresponding definition of $y_{\text {num }}^{\prime}(t)$ is

$$
y_{\text {num }}^{\prime}(t)= \begin{cases}\hat{P}_{1}(t), & t_{0} \leq t \leq t_{1} \\ \hat{P}_{2}(t), & t_{1} \leq t \leq t_{2} \\ \vdots & \\ \hat{P}_{n}(t), & t_{n-1} \leq t \leq t_{n} \\ \vdots & \end{cases}
$$

where $\hat{P}_{n}(t)$ not necessarily is the derivative of $P_{n}(t)$. The polynomials $P_{n}(t)$ and $\hat{P}_{n}(t)$ are commonly called local interpolants and can be of many types. The main requirements of the local interpolants are that they be sufficiently accurate and have sufficient continuity.

We write the norm of the estimated global error in the position at time $t$ as

$$
E_{r}(t)=\left\|y_{\text {num }}(t)-y_{\text {ref }}(t)\right\|_{2}
$$

and the norm of the estimated end-point global error as

$$
E_{r, \text { end }}\left(t_{f}\right)=\left\|y_{\text {num }}\left(t_{f}\right)-y_{\text {ref }}\left(t_{f}\right)\right\|_{2},
$$

where $t_{f}$ is the time at the end-point. The maximum of the norm of the estimated global error in position, loosely referred to as the maximum global error, is defined as the maximum norm of $E_{r, \max }(t)$ evaluated at $k$ evenly spaced sample points over each integration timestep. That is, we evaluate the maximum global error by introducing an accurate approximation $y_{\text {ref }}(t)$ to $y(t)$, sampling $\left\|y_{\text {num }}(t)-y_{\text {ref }}(t)\right\|_{2}$ over each time-step, and using the maximum over all sampled values to be the estimate of the maximum of $\left\|y_{\text {num }}(t)-y(t)\right\|_{2}$.

In way a similar to that for the position, the norm of the estimated global error in velocity at time $t$ and the maximum of this norm over the interval $\left[t_{0}, t_{f}\right]$ are defined as

$$
\begin{aligned}
& E_{v}(t)=\left\|y_{\text {num }}^{\prime}(t)-y_{\text {ref }}^{\prime}(t)\right\|_{2}, \\
& E_{v, \max }(t)=\max _{t \in\left[t_{0}, t_{f}\right]}\left\|y_{\text {num }}^{\prime}(t)-y_{\text {ref }}^{\prime}(t)\right\|_{2},
\end{aligned}
$$

where $y_{\text {num }}^{\prime}(t)$ and $y_{\text {ref }}^{\prime}(t)$ are vectors at any time $t$ of the derivatives to the numerical and reference solutions, respectively.

We store the pieces of information, like positions and times, in separate files. Meanwhile, in a post-processing program, we estimate the maximum global error by sampling the norm at $k$ evenly spaced data points on every sub-interval $\left[t_{n-1}, t_{n}\right]$ with respect to the reference solution, which we obtain at these stored values of time by forcing the integrator to hit the sample points, and then taking the maximum of these norms. To see the effect of different values of $k$ on the estimated maximum global error in position, we performed experiments using the combination of ERKN101217 and the 29stage interpolant, which are described in Section III A. The integration was performed over one million years of integration for the Jovian problem with local error tolerances $10^{-08}, 10^{-10}, 10^{-12}, 10^{-14}$, and $10^{-16}$. We found that $k=10$ led to an estimated maximum global error that was sufficiently accurate for our work; this is illustrated by the results in Table 1 . The rows labelled $k=50$ and $k=100$ list the percentage changes in the estimated maximum global error when compared with the values in row labelled $k=10$.

We observe from Table 1 that the data for $T O L=$ $10^{-08}$ shows a percentage change of zero. In this case, the estimated maximum global error occurs at $t_{f}$ and this is possible with $N$-body simulations; if this occurs, the percentage difference between different values in $k$ 


\section{S. REHMAN}

would be zero. We repeated the same set of experiments with other combinations. For example, when using the integrator $O D E X 2$ with its interpolant, described in Section IIIB, an increase of $k$ from 10 to 100 changed the estimated maximum global error by not more than 1\%. Throughout the remainder of this paper, all maximum global errors are estimated by sampling at 10 evenly spaced data points at every time-step.

\begin{tabular}{|c|c|c|c|c|c|}
\hline TOL & $10^{-08}$ & $10^{-10}$ & $10^{-12}$ & $10^{-14}$ & $10^{-16}$ \\
\hline$k=10$ & $4.70 \times 10^{-1}$ & $1.63 \times 10^{-3}$ & $4.82 \times 10^{-5}$ & $2.42 \times 10^{-5}$ & $8.80 \times 10^{-6}$ \\
\hline$k=50$ & $0 \%$ & $3.4 \times 10^{-2} \%$ & $3.9 \times 10^{-3} \%$ & $1.0 \times 10^{-2} \%$ & $5.2 \times 10^{-5} \%$ \\
\hline$k=100$ & $0 \%$ & $3.5 \times 10^{-2} \%$ & $3.9 \times 10^{-3} \%$ & $1.0 \times 10^{-2} \%$ & $4.2 \times 10^{-5} \%$ \\
\hline
\end{tabular}

Table 1. The values of estimated maximum global error using ERKN101217 with 29-stage interpolant obtained with different values of $k$ over one million years of integration for the Jovian problem with local error tolerances $10^{-08}, 10^{-10}, 10^{-12}, 10^{-14}$, and $10^{-16}$. The row labelled $k=10$ shows errors, whereas the rows labelled $k=50$ and $k=100$ list the percentage changes.

Usually, physical systems have conserved quantities, such as, total energy, total angular momentum, position and velocity of the center of mass of the bodies. Normally, these physical quantities are not conserved exactly by the numerical approximation and this deviation provides an insight about the accuracy of the numerical approximation. The total energy for a system of $N$ bodies interacting with one another through Newtonian forces is defined as

$H(t)=\frac{1}{2} \sum_{i=1}^{N} m_{i}\left(v_{i, \mathrm{num}}(t) \cdot v_{i, \mathrm{num}}(t)\right)-\sum_{j=1}^{N-1} \sum_{i=j+1}^{N} G \frac{m_{i} m_{j}}{d_{i j}(t)}$,

where $G$ is the gravitational constant, $m_{i}$ the mass of the $i^{\text {th }}$ body, $v_{i, \text { num }}(t)$ the numerical approximation to velocity of the $i^{\text {th }}$ body (components $3 i-2$ to $3 i$ of $\left.y_{\text {num }}^{\prime}(t)\right)$, and $d_{i j}(t)=\left\|r_{i, \text { num }}(t)-r_{j, \text { num }}(t)\right\|_{2}$ is the distance between the $i^{\text {th }}$ and $j^{\text {th }}$ bodies. Here, $r_{i, \text { num }}(t)$ is formed by the components $3 i-2$ to $3 i$ of $y_{\text {num }}(t)$ and $r_{j, \text { num }}(t)$ by the components $3 j-2$ to $3 j$ of $y_{\text {num }}(t)$, which represent the numerical approximations to the positions of the $i^{\text {th }}$ and $j^{\text {th }}$ bodies, respectively. The relative error in energy is defined as

$$
H_{\mathrm{rel}}(t)=\left|\frac{H_{0}-H(t)}{H_{0}}\right|,
$$

where $H_{0}$ is the total energy at $t=t_{0}$. The value of $\mu=G m$ is usually known more accurately than the value of $m$. Therefore, we use

$$
H_{\mathrm{rel}}(t)=\left|\frac{G H_{0}-G H(t)}{G H_{0}}\right| .
$$

The total angular momentum is defined as

$$
L(t)=\sum_{i=1}^{N} m_{i} r_{i, \mathrm{num}}(t) \times v_{i, \mathrm{num}}(t) .
$$

We define the relative error of the angular momentum as

$$
L_{\mathrm{rel}}(t)=\frac{\left\|L_{0}-L(t)\right\|_{2}}{\left\|L_{0}\right\|_{2}},
$$

where $L_{0}$ is the angular momentum at the initial time $t=t_{0}$.

\section{CONTINUOUS APPROXIMATIONS}

The well-known one-step methods for the numerical approximation of a system of ordinary differential equations are normally formulated such that numerical approximations are produced on mesh points $t_{0}<t_{1}<$ $t_{2}<\ldots<t_{f}$, which are determined by the step-size selection strategy of these numerical methods. As soon as a numerical approximation of $y_{\text {true }}(t)$ is required where $t$ is not at one of the mesh points, the numerical approximation must be extended to a continuous approximation. Pioneering work on continuous approximations for Runge-Kutta methods has appeared in the literature [11], and also for explicit methods [13]. This work was extended by others; see, for example, [1, 3-6, 10, 15, 16].

Figure 1 shows a local segment of the numerical solution $y_{\text {num }}$ over the time interval $\left[t_{n-1}, t_{n}\right]$. This solution is obtained by interpolation over the data calculated from the local problem (4). The local solution to (4) has a local error that is 0 at $t=t_{n-1}$. In contrast, the global error is not 0 for any $t \in\left[t_{n-1}, t_{n}\right]$. There are two contributions to the global error. First, the interpolation polynomial is not formed using true data. Rather, it is using an approximation at the end of the time-step. Second, even if it is true data then there is an interpolation error at the intermediate times $t \in\left[t_{n-1}, t_{n}\right]$. If we denote the estimated global error in an interpolated value on $\left[t_{n-1}, t_{n}\right]$ by $E_{\mathrm{r}, \text { int }}(t)$, then the relative error with respect to the estimated end-point global error is

$$
\begin{aligned}
R_{\mathrm{err}}(t) & =\frac{\text { Estimated global error in an interpolated value on }\left[t_{n-1}, t_{n}\right]}{\text { Estimated global error in the numerical solution at } t_{n}} \\
& =\max _{t \in\left[t_{n-1}, t_{n}\right]} \frac{E_{\mathrm{r}, \text { int }}(t)}{E_{\mathrm{r}, \text { end }}\left(t_{n}\right)} .
\end{aligned}
$$

If the integration error dominates the interpolation error, then the relative error $R_{\text {err }}(t)$ tends to 1 as $t \mapsto \infty$. 


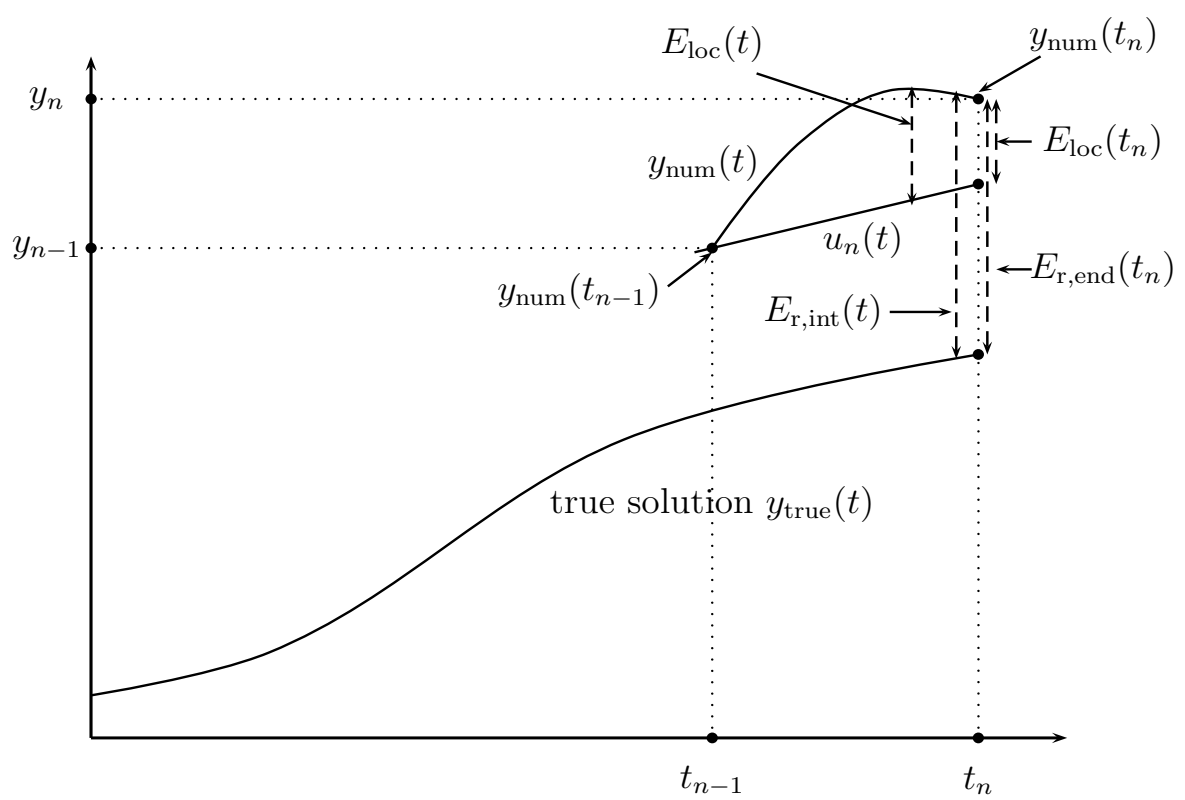

Fig. 1. Classification of the errors when using a numerical integrator together with an interpolation polynomial

\section{A. Continuous approximation with embedded RKN methods}

Dormand and Prince [3] and then Baker et al. [1] constructed a continuous approximation for embedded RKN pairs, where a third RKN process of order $p^{*}$ is used for the numerical approximations of $y\left(t_{n-1+\alpha}\right)$ and $y^{\prime}\left(t_{n-1+\alpha}\right)$ with $\alpha$ typically in $(0,1]$. These approximations are usually called interpolants and are denoted by $y_{n-1+\alpha}^{*}$ and $y_{n-1+\alpha}^{\prime *}$ :

$$
\begin{aligned}
& y_{n-1+\alpha}^{*}=y_{n-1}+\alpha h_{n-1} y_{n-1}^{\prime}+\alpha^{2} h_{n-1}^{2} \sum_{i=1}^{s^{*}} b_{i}^{*}(\alpha) k_{i}^{*}, \\
& y_{n-1+\alpha}^{\prime *}=y_{n-1}^{\prime}+\alpha h_{n-1} \sum_{i=1}^{s^{*}} b_{i}^{\prime *}(\alpha) k_{i}^{*}
\end{aligned}
$$

where,

$$
\begin{aligned}
k_{i}^{*} & =f\left(t_{n-1}+c_{i}^{*} \alpha h_{n-1}, y_{n-1}+c_{i}^{*} \alpha h_{n-1} y_{n-1}^{\prime}\right. \\
& \left.+\alpha^{2} h_{n-1}^{2} \sum_{j=1}^{i-1} a_{i j}^{*} k_{j}^{*}\right), \quad i=1, \ldots, s^{*}
\end{aligned}
$$

If $s^{*}=s$, then no extra function evaluations are required for $y_{n-1+\alpha}^{*}$ and $y_{n-1+\alpha}^{* *}$. As before, the prime in $b_{i}^{\prime *}$ is purely notational and does not involve the differentiation operator. The approximation to $y_{\text {num }}(t)$ is continuous and has continuous first- and second-derivatives, because it interpolates $y, y^{\prime}$, and $y^{\prime \prime}$ at both ends of each timestep. For ERKN methods, the derivative interpolants can be derived separately, and not as the derivative to the solution interpolants themselves.

For ERKN101217 integrator, we have used 23-stage, 26 -stage, and 29-stage interpolants with $p^{*}=10, p^{*}=$ 11 , and $p^{*}=12$, respectively. The coefficients for these interpolants are freely available on-line [1]. For ERKN689 integrator, we have used a 12-stage interpolant with $p^{*}=8$. Note that the coefficients for the interpolant for $y$ and $y^{\prime}$ of ERKN689 integrator are provided (private communication) by P. W. Sharp.

\section{B. Continuous approximation with $O D E X \mathscr{2}$}

The ODEX2 integrator has a solution interpolant. We added a derivative interpolant by differentiating the solution interpolant. The solution interpolant provides an approximation to the $i^{\text {th }}$-component of the solution at time $t$. The polynomial $P_{\mu}(\alpha)$ for the solution interpolant can be written as

$$
\begin{aligned}
P_{\mu}(\alpha) & =y_{(i)}+\alpha y_{(M+i)}+\alpha(1-\alpha) y_{(2 M+i)}+\alpha^{2}(1-\alpha) y_{(3 M+i)}+\alpha^{3}(1-\alpha)^{2} y_{(4 M+i)} \\
& +\alpha^{2}(1-\alpha)^{3} y_{(5 M+i)}+\left(\alpha-\alpha^{2}\right)^{3} \sum_{j=0}^{k} \frac{\left(\alpha_{1}\right)^{j}}{j !} y_{(M(5+j)+i)},
\end{aligned}
$$

where $\alpha_{1}=\left(t-t_{n-1}\right) / h-0.5$ and $M$ is the number of ordinary differential equations. The degree of $P_{\mu}(\alpha)$ is $\mu+4$, with $-1 \leq \mu \leq 2 k$, where $k$ is related to the integration order. 


\section{S. REHMAN}

The polynomial used for the continuous approximation of the derivative provides an approximation to the $i^{\text {th }}$ component of the derivative at time $t$. The order of this derivative interpolant is one less than $P_{\mu}(\alpha)$ and can be written as

$$
\begin{aligned}
P_{\mu}^{\prime}(\alpha) & =\frac{1}{h}\left[y_{(M+i)}+(1-2 \alpha) y_{(2 M+i)}+\left(2 \alpha-3 \alpha^{2}\right) y_{(3 M+i)}+\alpha^{2}(1-\alpha)(3-5 \alpha) y_{(4 M+i)}\right. \\
& \left.+\alpha(1-\alpha)^{2}(2-5 \alpha) y_{(5 M+i)}+\left(\alpha-\alpha^{2}\right)^{3} \sum_{j=1}^{k} \frac{\left(\alpha_{1}\right)^{j-1}}{(j-1) !} y_{(M(5+j)+i)}+3\left(\alpha-\alpha^{2}\right)^{2}(1-2 \alpha) \sum_{j=0}^{k} \frac{\left(\alpha_{1}\right)^{j}}{j !} y_{(M(5+j)+i)}\right]
\end{aligned}
$$

\section{NUMERICAL TESTING FOR LONG-TERM} SIMULATIONS

First of all, we discuss error growth in position and velocity by using the appropriate combinations integrators: ODEX2, ERKN689, and ERKN101217, and interpolation schemes over one million years for the Jovian problem.

We have obtained the reference solution in quadrupleprecision using the combination of ERKN101217 integrator and the 29-stage interpolant with $T O L=$ $10^{-18}$. To quantify this particular choice for the reference solution, we also integrated the Jovian problem using the combination of ERKN101217 and the 29-stage interpolant with $T O L=10^{-20}$. It has been observed that the maximum difference between positions and velocities of these two solutions is no more than $4.6 \times 10^{-13}$. It has also been observed that in most cases, the maximum of the global error occurs at the end-point of the integration. We also integrated the Jovian problem in quadrupleprecision using the combination of ERKN689 and the 12-stage interpolant with the tolerance TOL $=10^{-18}$ and observed that the maximum difference with the solution for the combination of ERKN101217 and the 29-stage interpolant with $T O L=10^{-18}$ is no more than $5.1 \times 10^{-13}$. This suggests that the combination of ERKN101217 and the 29-stage interpolant with $T O L=10^{-18}$ is fairly accurate to acquire the reference solution.

We have performed numerical experiments to observe the unweighted $L_{2}$-norm of the estimated maximum global error in the position as a function of tolerance with three different combination; ODEX2 and its built-in interpolant, ERKN689 and the 12-stage interpolant, and ERKN101217 and the 29-stage interpolant over one million years for the Jovian problem. It has been observed that the maximum global error obtained with the combination of $O D E X 2$ and its built-in interpolant is ranging from $8.0 \times 10^{-5}$ to $1.1 \times 10^{2}$. The best observed accuracy $8.0 \times 10^{-5}$ is obtained with $T O L=10^{-16}$ and the minimum accuracy $1.1 \times 10^{2}$ with $T O L=10^{-08}$. The combination of ERKN101217 and the 29-stage interpolant is an accuracy that ranges from $8.8 \times 10^{-6}$ to $4.7 \times 10^{-1}$. The best observed accuracy is obtained with $T O L=10^{-16}$ and the minimum accuracy with $T O L=10^{-08}$. Similarly, the combination of ERKN689 and the 12-stage interpolant has an accuracy ranging from $9.8 \times 10^{-7}$ to
$4.4 \times 10^{-2}$, with the best observed accuracy at $T O L=$ $10^{-14}$ and the minimum accuracy is obtained at $T O L=$ $10^{-08}$.

To test the analysis that led to the definition of the error $R_{\text {err }}(8)$, we integrate the Jovian problem over one million years using all the integrators and interpolation schemes described so far. On every accepted integration time-step, we obtain the $L_{2}$-norm of $H_{\text {rel }}$ and $L_{\text {rel }}$ at 10 evenly spaced values of time. The maximum of these 10 error values is taken as the maximum error (care has been taken to make efficient use of storage) on the integration time-step. The $\bar{S}-13$ integrator uses a fixed step-size of 4 days, and we set the tolerances to $10^{-16}, 10^{-11}$, and $10^{-10}$ for ODEX2, ERKN101217, and $E R K N 689$, respectively (the variation of tolerance is subject to achieving a maximum global error of $10^{-4}$ with variable-step-size integrators).

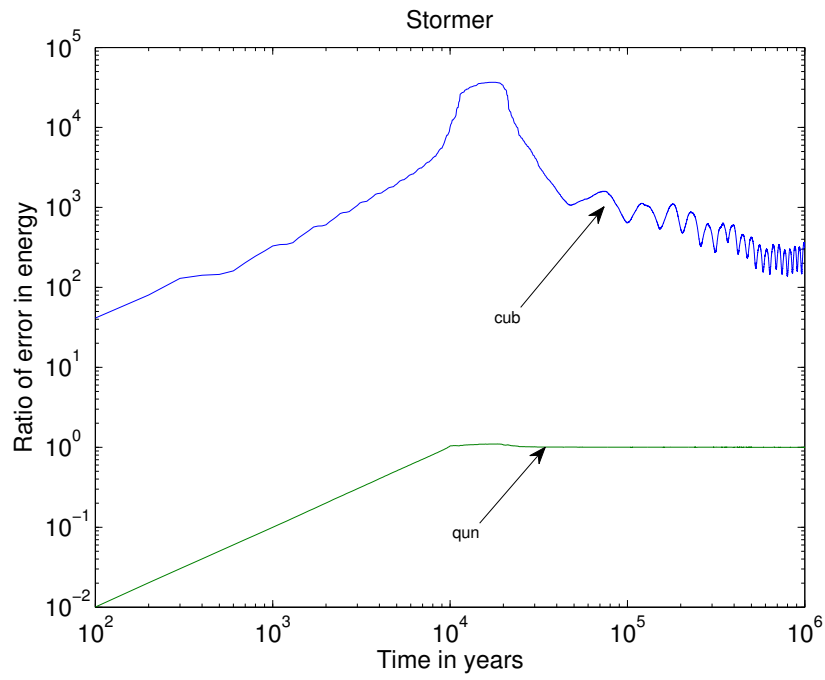

Fig. 2. The ratio of the maximum $H_{\text {rel }}$ at the interior points to the end-point (of a step) $H_{\text {rel }}$ for the $\bar{S}-13$ integrator using cubic and quintic Hermite interpolations over one million years for the Jovian problem

Figure 2 depicts the ratio of the maximum $H_{\text {rel }}$ at the interior points to the end-point $H_{\text {rel }}$ for the $\bar{S}-13$ integrator using cubic and quintic interpolations. We observe from the plot for the cubic Hermite interpolation polynomial that $R_{\text {err }}$ appears bounded from above by $5 \times 10^{4}$ for small $t$ and then gradually decreases to no more than $6 \times 10^{2}$ at $10^{6}$ years. The large value 
of $R_{\text {err }}$ may be due to the fact that the interpolation polynomial used for the velocity components is only of order 2. The plot for the quintic Hermite interpolation polynomial shows that the relative error in energy is approximately 1 for the entire interval of integration. This verifies the result of Grazier et al. [7] that, when the time-step is chosen so that the $\bar{S}-13$ methods satisfy Brouwer's law, the one-step quintic Hermite interpolation is sufficiently accurate. A similar behaviour with cubic and quintic polynomials is observed for the relative error in angular momentum.

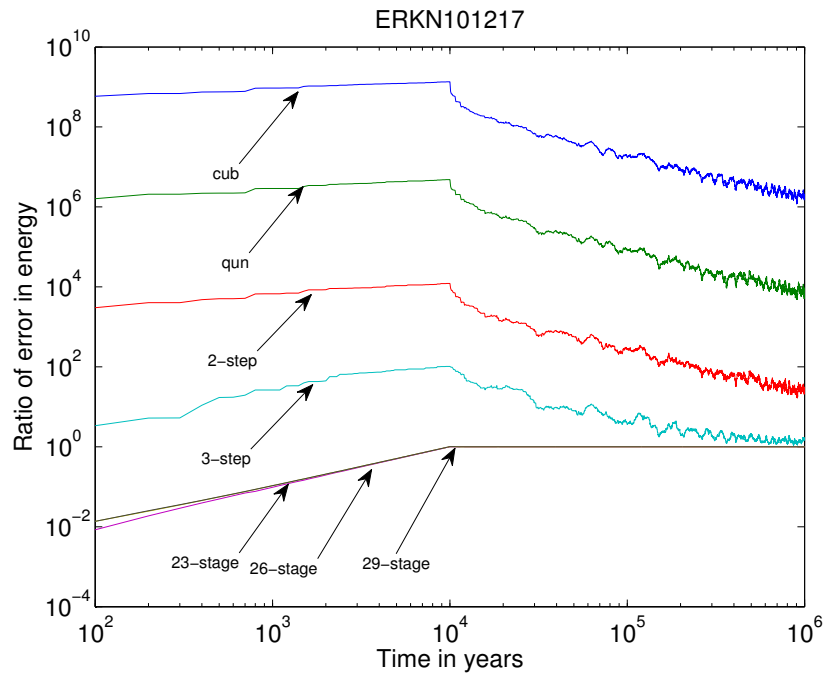

Fig. 3. The ratio of the maximum $H_{\text {rel }}$ at the interior points to the end-point $H_{\text {rel }}$ for ERKN101217 using cubic, quintic, 2-step, 3-step interpolations, and 23-stage, 26-stage, 29-stage interpolants over one million years of integration for the Jovian problem with local error tolerance of $10^{-11}$

Figure 3 represents the ratio of the maximum $H_{\text {rel }}$ at the interior points to the end-point $H_{\text {rel }}$ for ERKN101217 using cubic, quintic, 2-step and 3-step interpolations [17], which are of degrees $3,5,8$, and 11 , respectively; and 3 interpolants of 23,26 , and 29 stages, having orders 10 , 11 , and 12 , respectively. We observe for the low-order interpolation schemes, i.e., cubic, quintic, and two-step, that $R_{\text {err }}$ in energy does not decrease to 1 at the end of $10^{6}$ years. With all three interpolants the $R_{\text {err }}$ is approximately 1 for the entire interval of integration. We also observe that the 3 -step interpolation polynomial achieves the same accuracy (with $R_{\text {err }}$ in energy approximately 1 ) at the end of the integration, although initially it is dominated by the interpolation error. Therefore, until about $10^{4}$ years, it is better to use one of the interpolants if the smallest error is required. However, if the required integration time is large enough, the integration error starts dominating and then it is better to switch to a 3 -step interpolation polynomial because this is going to save on CPU-time and does not sacrifice accuracy.

We also performed experiments measuring $R_{\text {err }}$ for the energy and angular momentum for ERKN689 using the cubic, quintic, and two-step Hermite interpolation schemes, and together with its 12-stage interpolant; and for $O D E X 2$ using cubic, quintic, two-step and three-step interpolations, and together with the interpolant. For ERKN689, the two-step interpolation scheme and the 12-stage interpolant achieved a ratio of approximately 1. For $O D E X 2$, the only continuous approximation that achieved the same accuracy was the interpolant and its derivative that comes with $O D E X 2$.

To observe the behaviour of conserved quantities like energy and angular momentum using different interpolation schemes with different integrators, we performed many experiments. All these experiments were done for the Jovian problem over up to $10^{8}$ years. One of the strong observations about all of these experiments that applies except when using the quintic Hermite interpolation scheme with the $\bar{S}-13$ integrator is as follows: if a low-order interpolation scheme is used with a high-order integrator then the interpolation error at a particular time will dominate the integration error and the total error will not increase with $t$. This is because the interpolation error does not grow with time. For example, if a 12-th order integrator is used with cubic Hermite interpolation, then the total error is given by

$$
\begin{aligned}
\text { Total error } & =C t^{3 / 2} h^{12}+D h^{4} \\
& \approx D h^{4}, \quad C, D=\text { Constant },
\end{aligned}
$$

provided $t$ is not too large and $C$ is not significantly larger than $D$. On the other hand, if the interpolation polynomial and the integrator are of the same order, then the total error will increase with the passage of time.

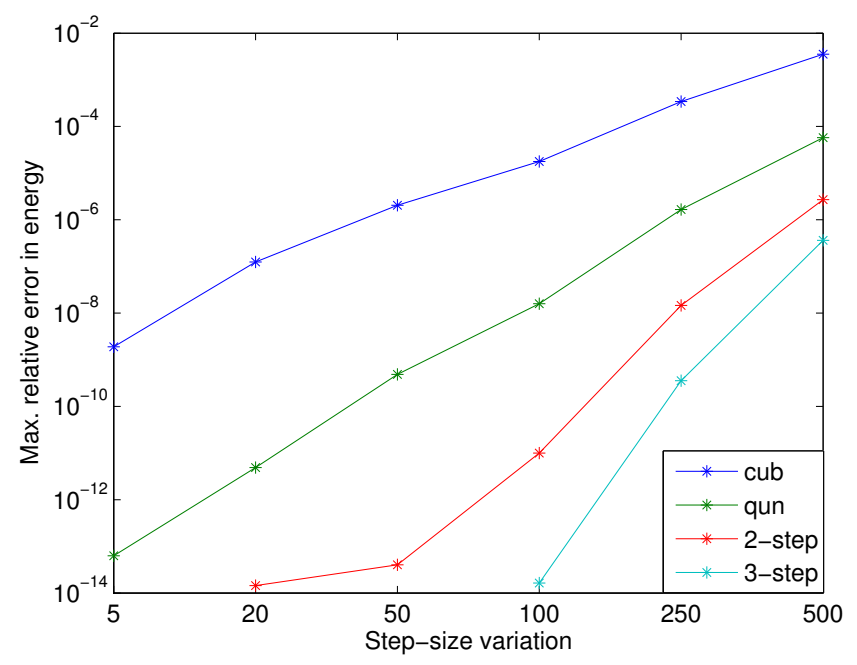

Fig. 4. The maximum $H_{\text {rel }}$ for the cubic, quintic, 2-step, and 3 -step interpolations with different step-size sequences over one period of Jupiter for the Jovian problem

We now want to observe the effect of the step-size on $H_{\text {rel }}$ and $L_{\text {rel }}$ while performing long-term simulations using different interpolation schemes with different integrators. Therefore, we set up an experiment to anticipate the behaviour of this relative error; see Figure 4 . This experiment provides an insight about the interpolation error in energy and angular momentum. We integrate the Jovian problem using the ERKN101217 integrator over a time interval that equals one period of 


\section{S. REHMAN}

Jupiter. We obtain a reference solution by integrating in quadruple precision with $T O L=10^{-18}$ to eliminate the possible effect of integration error. We run six experiments, where we record position, velocity and acceleration at the end of every 5, 20,50,100, 250, and 500 days, by forcing the integrator to hit these time-points. The period of Jupiter is not divisible by 5, 10, 50, 100, 250 , and 500 and hence the last step of each integration was shorter than the previous steps. The selection of such step-size sequences is done in accordance with the average step-sizes taken by different integrators when integrating the Jovian problem. For example, over $10^{6}$ years, $\bar{S}-13$ takes a time-step of 4 days, while on average, over the same time interval, ERKN689 with $T O L=10^{-10}, E R K N 101217$ with $T O L=10^{-11}$ and $O D E X 2$ with $T O L=10^{-16}$ take approximately 65,290 and 260 days, respectively.

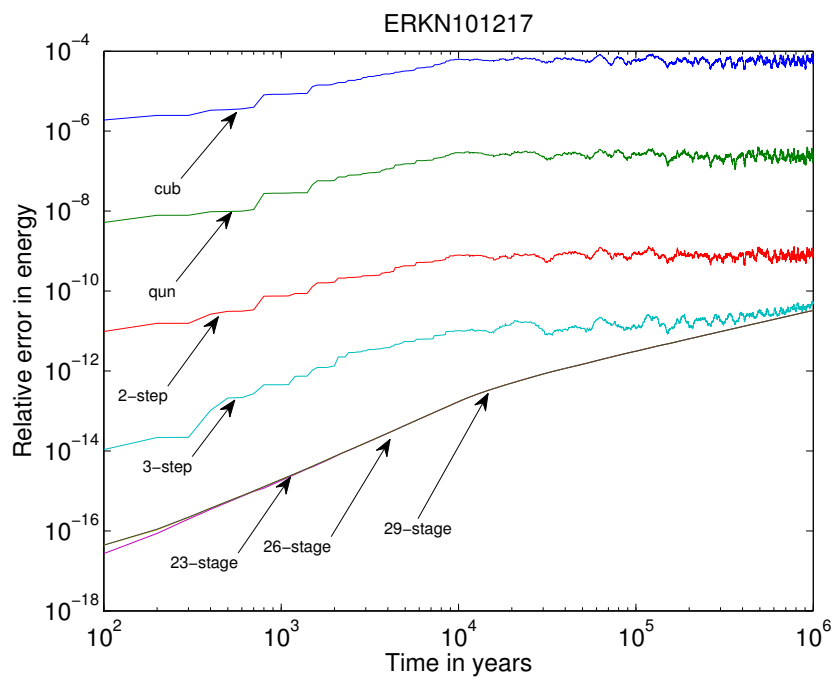

Fig. 5. The $H_{\text {rel }}$ for ERKN101217 using cubic, quintic, 2step, 3-step interpolations, and 23-stage, 26-stage, 29-stage interpolants over one million years of integration for the Jovian problem with the local error tolerance of $10^{-11}$

For different step-size sequences, we use appropriate interpolation schemes; for example, as discussed earlier, the 3-step interpolation scheme needs not to be used for the $\bar{S}-13$ integrator with a step-size of 4 days, since the quintic Hermite interpolation is sufficient. Then on each step-size, we use interpolation and sample the solution at 10 equally-spaced data points, from which we determine the local maxima for $H_{\text {rel }}$ and $L_{\text {rel }}$. For each interpolation scheme, we observe that there is a considerable variation in $H_{\text {rel }}$ and $L_{\text {rel }}$ as a function of the step-size. For example, the biggest variation of approximately 9 orders of magnitude for $H_{\text {rel }}$ was observed with the quintic Hermite interpolation scheme over the range of 5 to 500 days step-size sequences. This particular example has a reasonably good agreement with the expected difference of approximately 10 orders of magnitude, because the expression for the relative error in energy is dominated by the velocity term of order 5 with a quintic interpolation. This means that, by using expression $\alpha h^{p+1} \frac{y_{\text {true }}^{(p+1)}(\xi)}{(p+1) !}$ for the interpolation error, the expected difference of 5 and 500 days step-size sequences is $(500 / 5)^{5}=10^{10}$.

Figure 5 contains the graphs of the relative error in energy for ERKN101217 when used with the cubic, quintic (one-step), 2-step and 3-step interpolation polynomials, of degrees $3,5,8$ and 11 , respectively; and the three interpolants of 23,26 , and 29 stages, having orders 10, 11, and 12, respectively. The ERKN101217 integrator for an integration of $10^{6}$ years using $T O L=$ $10^{-11}$ requires, on average, a step-size of approximately 290 days. We find that the total error for the cubic, quintic, and 2-step interpolations does not increase with $t$. It remains pretty much constant, as was expected, because the interpolation error dominates the integration error.

The interpolation errors at the end of the integration have a reasonably good agreement with the results obtained in Figure 4. With all three interpolants, i.e., 23-stage, 26-stage and 29-stage, we have obtained an accuracy of approximately $10^{-11}$. With the 3 -step interpolation scheme, which is cheaper than all three interpolants, the accuracy is about the same at the end of the integration, although initially it is dominated by the interpolation error. Therefore, it is better to use an interpolant if the best accuracy is required. However, if the time interval is large enough, about $10^{5}$ years or more, then the integration error starts to dominate and it is better to switch to a 3-step interpolation, because this will save CPU-time and does not affect accuracy. We also found (not shown) that the 2-step interpolation which requires less CPU-time than the 3-step interpolation will give the same accuracy after even longer integration times.

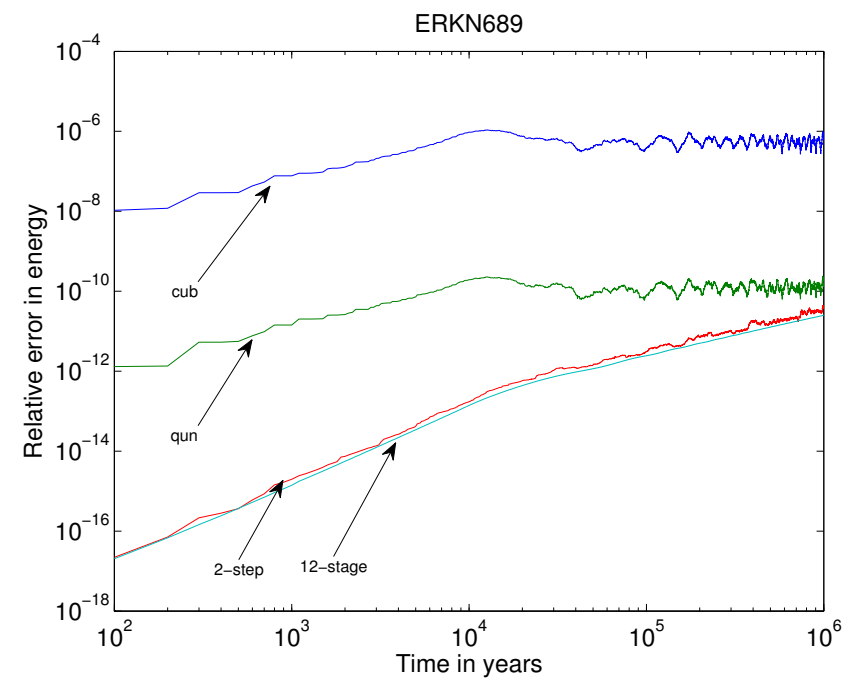

Fig. 6. The $H_{\text {rel }}$ for $E R K N 689$ using cubic, quintic, 2-step Hermite interpolations, and the 12-stage interpolant over one million years of integration for the Jovian problem with local error tolerance of $10^{-10}$

Figure 6 shows the relative error growth in energy for ERKN689 using the cubic, quintic, and two-step Hermite interpolation schemes together with a 12-stage interpolant. For $T O L=10^{-10}$, the average time-step is approximately 65 days. For the two-step interpolation and the 12-stage interpolant, there is no real difference 
between these two graphs, unlike for ERKN101217 using the 3 -step interpolation and its interpolants as shown in Figure 5. The two errors increase side by side right from the beginning, indicating that there is no sense in using the 12-stage interpolant, because it costs more CPU-time than the 2-step interpolation.

We extend the above experiment for ERKN689 by performing an integration of 10 million years. After approximately 7 million years (not shown) it is beneficial to switch to quintic interpolation, because it takes less CPU-time. The quintic Hermite interpolation is not under consideration for the ERKN101217 integrator, because the total error differs by three orders of magnitude in comparison with ERKN689.

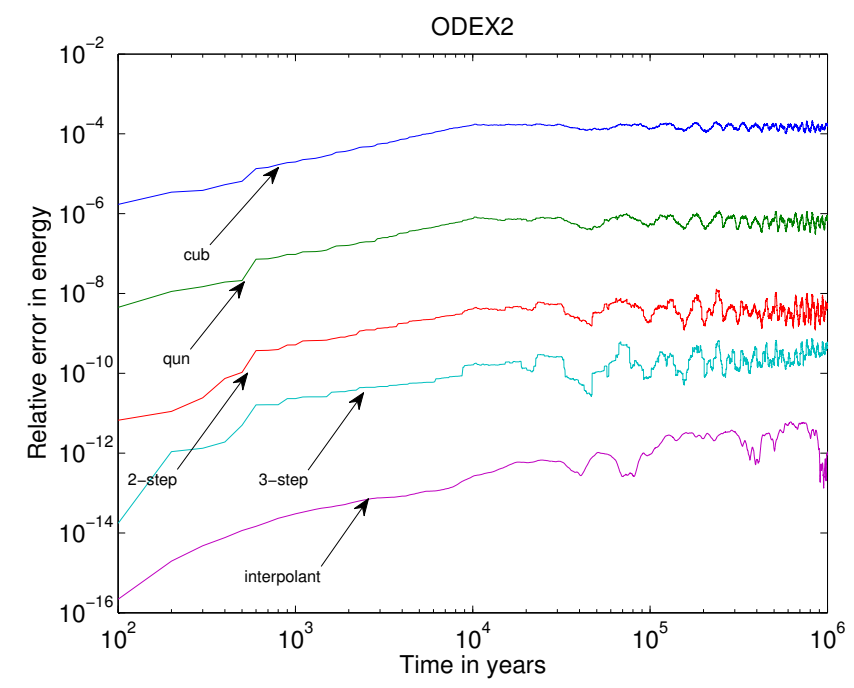

Fig. 7. The $H_{\text {rel }}$ for $O D E X 2$ using cubic, quintic, 2-step, 3step Hermite interpolations, and its built-in interpolant over one million years of integration for the Jovian problem with the local error tolerance of $10^{-16}$

Figure 7 illustrates the relative error growth in energy for $O D E X 2$ using cubic, quintic, two-step and three-step interpolations together with its interpolant. On average, $O D E X 2$ uses a time-step of approximately 260 days if we set $T O L=10^{-16}$; normally it is not recommended to take such a small tolerance, because it is very close to the machine precision and the round-off error could affect the results greatly. We observe that the graph for the interpolant shows oscillations, which indicates that the round-off error is significant. We also found that there are quite a few rejected time-steps. Combined with the asence of any rejected time-steps with either of the tolerances $10^{-14}$ and $10^{-15}$, this is further evidence that the results for $T O L=10^{-16}$ are affected by the round-off error. Nevertheless, we are gaining an accuracy of approximately $10^{-11}$ (determined by a linear least square fit using data obtained by continuous approximation). This accuracy for $T O L=10^{-16}$ is approximately one order of magnitude better than the accuracy obtained with $T O L=10^{-15}$, which is expected when reducing the tolerance by a factor of 10 . This observation suggests that the round-off error is insignificant. The behaviour of the interpolant at the end of the integration shows an abrupt dip followed by rapid oscillations. We investigated this further by performing the experiments with the same tolerance for up to $10^{8}$ years. While the oscillations continue, both $H_{\text {rel }}$ and $L_{\text {rel }}$ increase with the passage of time and the curve ends at a total error around $10^{-9}$ and $10^{-10}$, respectively.

We performed the same experiments using the $\bar{S}-13$ integrator with a step-size of four days, along with the cubic and quintic Hermite interpolation schemes. We found for the cubic interpolation that the total error in energy and angular momentum does not grow with $t$, but the maximum global error has a reasonably good agreement with the results obtained using a step-size sequence of five days in the experiments (see Figure 4). For the quintic Hermite interpolation, the error growth in energy and angular momentum is below linear.

\begin{tabular}{|l|l|c|c|c|c|}
\hline Integrator & Interpolation & $E_{r}$ & $E_{v}$ & $H_{\text {rel }}$ & $L_{\text {rel }}$ \\
\hline$E R K N 689$ & - & 1.980 & 1.987 & 0.986 & 0.971 \\
\hline$E R K N 689$ & 2 -step & - & - & 0.991 & 0.979 \\
\hline$E R K N 689$ & 12 -stage & - & - & 0.985 & 0.970 \\
\hline ERKN1012 & - & 1.997 & 1.996 & 0.998 & 1.005 \\
\hline$E R K N 101217$ & 3 -step & - & - & 0.677 & 0.683 \\
\hline$E R K N 101217$ & 23 -stage & - & - & 0.998 & 1.005 \\
\hline$E R K N 101217$ & 26 -stage & - & - & 0.997 & 1.004 \\
\hline$E R K N 101217$ & 29 -stage & - & - & 0.997 & 1.005 \\
\hline$O D E X 2$ & - & 1.514 & 1.457 & 0.327 & 0.474 \\
\hline$O D E X 2$ & 3 -step & - & - & 0.589 & 0.632 \\
\hline$O D E X 2$ & interpolant & - & - & 0.325 & 0.465 \\
\hline $\bar{S}$-13 & - & 1.377 & 1.542 & 0.689 & 0.718 \\
\hline $\bar{S}$-13 & quintic & - & - & 0.687 & 0.724 \\
\hline
\end{tabular}

Table 2. The exponent $b$ of the power law for the global error in position and velocity, and $H_{\text {rel }}$ and $L_{\text {rel }}$ over one million years for the Jovian problem. We used local error tolerances $10^{-11}, 10^{-10}$, and $10^{-16}$ with different interpolation schemes, for the integrators ERKN689, ERKN101217, ODEX2, and $\bar{S}$ -

13 , respectively. The step-size for $\bar{S}-13$ is four days

Table 2 shows the exponent $b$ in a linear least squares fit for the power law $a t^{b}$. In particular, when loworder interpolation schemes are used with high-order variable-step-size integrators, the exponent $b$ of the power law should be very close to zero. For example, for ERKN689, using the cubic and quintic Hermite interpolation schemes, the exponent $b$ varies from 0.029 to 0.35 for $H_{\text {rel }}$ and $L_{\text {rel }}$. We already noted that the ODEX2 integrator is affected by round-off error if TOL $=10^{-16}$. To observe the extended behaviour of $H_{\mathrm{rel}}$ and $L_{\mathrm{rel}}$, we integrated $O D E X 2$ using $T O L=10^{-16}$ over $10^{7}$ years and observed the values for $H_{\text {rel }}$ and $L_{\text {rel }}$ as 0.76 and 0.89 , respectively. We also repeated experiments for $O D E X 2$ with $T O L=10^{-15}$ and $10^{-14}$ and observed that the value of the exponent increases. In particular, with $T O L=10^{-14}$, the value of $b$ is approximately 1.92 for the global error in position; for $H_{\text {rel }}$ and $L_{\text {rel }}$, it is approximately 0.99 and 1.01 , respectively. 


\section{S. REHMAN}

\section{SUMMARY AND CONCLUSIONS}

The main objective of this paper was to investigate the possibilities of replacing the interpolants of certain integrators by other interpolation schemes, perhaps at a cost of a little bit of accuracy. We analysed and compared the error growth for different combinations of numerical integrators and interpolation schemes. Our numerical testing involved comparing combinations of different integrators and interpolation schemes over a short time interval and several long time intervals, as long as 100 million years for the Jovian problem with local error tolerances ranging from $10^{-16}$ to $10^{-08}$.

The interpolation schemes play a vital role in our work. The low-order interpolation schemes are unlikely to be used in practice. We have included them in our testing because we wanted to assess the effectiveness of these interpolation schemes. Especially, it makes sense to use a low-order interpolation scheme with a highorder Störmer method when the step-size is four days (for the Jovian problem), because this choice means the interpolation error is below machine precision. We performed many experiments using different interpolation schemes with different integrators both over a short time interval and several long time intervals of duration as long as 100 million years. For long term simulations, experiments were performed for the Jovian problem integrated up to $10^{8}$ years. One of the strongest conclusions from these experiments is that the order of the continuous approximation should, with one notable exception in this paper, be compatible with the order of the integrator. The notable exception occurs for the higher order Störmer methods when used with small step-sizes. The order of the continuous approximation can be significantly lower than the order of the Störmer method. The reason why we get away with the low-order continuous approximation is because the $\bar{S}-13$ integrator uses an artificially small step-size of four days to eliminate the truncation error at the machine precision and we are just left with the round-off error. However, if we want the truncation error to be $10^{-16}$, then we might use the step-size of sixteen days rather than four days. When we use sixteen days, then the low-order continuous approximation (quintic Hermite interpolation) would fail.

To observe the behaviour of conserved quantities like energy and angular momentum using different interpolation schemes with different integrators, quite a few experiments were performed. All these experiments were performed for the Jovian problem over upto $10^{8}$ years. One of the strong observations about all these experiments is that if a low order interpolation scheme is used with a high order integrator then the interpolation error for a particular time will dominate the integration error and will not increase with $t$. This is because the interpolation error doesn't grow with time. We investigated the relative error in energy for $E R K N 101217$ when used with cubic, quintic, twostep and three-step interpolation polynomials and the 3 interpolants, for ERKN689 using the cubic, quintic, and two-step Hermite interpolation schemes together wi- th a 12-stage interpolant, for $O D E X 2$ using cubic, quintic, two-step and three-step interpolations together with the interpolant, and for $\bar{S}-13$ using cubic, and quintic interpolation schemes.

ERKN101217 across the integration of $10^{6}$ years using $T O L=10^{-11}$ on average takes approximately 290 days of time-step. We observed that the total error for all the low order interpolation schemes doesn't increase with the passage of time. With all three interpolants, we obtained the accuracy of approximately $10^{-11}$ and then with the 3 -step interpolation scheme which is cheaper than all the three interpolants, the accuracy is about the same at the end of the integration, though initially it was dominated by the an interpolation error. So, about $10^{4}$ years out of all these possibilities, it is better to use interpolant if the maximum accuracy is required but if it is integrated far enough, the integration error starts dominating, and then it is better to switch to a 3-step interpolation because that is going to save the CPU time and doesn't cost the accuracy. And if integrated even further, a 2-step interpolation is going to give the same accuracy, which is a little cheaper, than a 3 -step interpolation.

ERKN689 with TOL $=10^{-10}$ uses an average timestep of approximately 65 days. We observed that for a two-step interpolation and a 12-stage interpolant, there is not any real difference between the plotted graphs for the relative error in energy, unlike ERKN101217 using a 3-step interpolation and interpolants, the two graphs go side by side right from the beginning, indicating right from the beginning there is no sense of using a 12-stage interpolant because it takes more CPU time in comparison with a 2-step interpolation. And after approximately 7 million years it is handy to switch to a quintic interpolation (which was not even under consideration with ERKN101217 as these two integrators with the quintic Hermite interpolation are different total error by three orders of magnitude) as it costs less CPU time.

On average, $O D E X 2$ with $T O L=10^{-16}$ uses a timestep of approximately 260 days. We observed that the graph for the interpolant shows oscillations, which is indicating the possible effect of the round-off error. We also observed that there are quite a few rejected timesteps. This combined with the absence of any time-steps with either of the tolerances $10^{-14}$ and $10^{-15}$ is further evidence that the results for $T O L=10^{-16}$ are being affected by the round-off error. Although, it is affected by the round off error, yet we are gaining accuracy up to $10^{-11}$, which is approximately one order of magnitude better than the accuracy obtained with $T O L=$ $10^{-15}$ and that is what you can expect by reducing the tolerance 10 times. So, reducing the tolerance from $10^{-15}$ to $10^{-16}$ is not making things difficult but we are gaining accuracy, which indicates that the round-off error is insignificant. For the interpolant, the behaviour of the plot at the end of the integration shows that the curve with oscillations is dipping-down. To observe the extended behavior we performed the experiment with the same tolerance for up to $10^{8}$ years and it was observed that (with the oscillation going up and down) both $H_{\text {rel }}$ and 
$L_{\text {rel }}$ increase with the passage of time and end-up with the total error around $10^{-9}$ and $10^{-10}$, respectively. For $\bar{S}-13$, the integration was performed in double precision and we observed that for cubic interpolation the total error in energy and angular momentum doesn't grow with $t$ but the achieved accuracy has a reasonably good agreement with the results obtained using 5 days in the experiment shown in Figure 4. For the quintic
Hermite interpolation, the error growth in energy and angular momentum has been observed below the linear (the exponent of power law is less than 1 as mentioned in Table 2) error growth.

Acknowledgment. We are grateful to reviewer for their valuable suggestions to improve the quality of the article.
[1] T. S. Baker, J. R. Dormand, P. J. Prince, App. Num. Math. 29, 171 (1999); https://doi.org/10.1016/S016 8-9274 (98) 00065-8.

[2] J. Dormand, M. E. A. El-Mikkawy, P. Prince, J. Numer. Anal. 7, 423 (1987); https://doi.org/10.1093/imanum /7.4.423.

[3] J. R. Dormand, P. J. Prince, Celest. Mech. 18, 223 (1978); https://doi.org/10.1007/BF01230162.

[4] J. R. Dormand, P. J. Prince, Comp. Math. Appl. 12A, 1007 (1986); https://doi.org/10.1016/0898-1221(86 ) $90025-8$.

[5] J. R. Dormand, P. J. Prince, Comp. Math. Appl. 13, 937 (1987); https://doi.org/10.1016/0898-1221(87 ) $90066-6$.

[6] W. H. Enright, K. R. Jackson, S. P. Norsett, P. G. Thomsen, ACM Trans. Math. Softw. 12, 193 (1986); https://doi.org/10.1145/7921.7923.

[7] K. R. Grazier, W. I. Newman, P. W. Sharp, Astron. J. 145, 112 (2013): https://doi.org/10.1088/0004-625 $6 / 145 / 4 / 112$

[8] K. R. Grazier, W. I. Newman, J. M. Hyman, P. W. Sharp, ANZIAM J. 46, C1086 (2005); https://doi.or g/10.21914/anziamj.v46i0.1008.

[9] K. R. Grazier, W. I. Newman, W. M. Kaula, J. M. Hyman, Icarus 140, 341 (1999); https://doi.org/10.1 006/icar.1999.6146.

[10] E. Hairer, S. P. Nørsett, G. Wanner, Solving Ordinary Differential Equations I: Nonstiff Problems (SpringerVerlag, 1987).

[11] M. K. Horn, SIAM J. Num. Anal. 20, 558 (1983); https : //doi.org/10.1137/0720036.

[12] E. J. Nyström, Acta Soc. Sci. Fennicae 50, 1 (1925); https://lib.ugent.be/catalog/rug01:001785116.

[13] L. F. Shampine, SIAM J. Num. Anal. 22,1014 (1985); https://doi.org/10.1137/0722060.

[14] C. Störmer, Radium (Paris) 9, 395 (1912); https://do i.org/10.1051/radium:01912009011039501.

[15] Ch. Tsitouras, G. Papageorgiou, Computing 43, 255 (1990); https://doi .org/10.1007/BF02242920.

[16] J. H. Verner, SIAM J. Numer. Anal. 30, 1446 (1990); https://doi.org/10.1137/0730075.

[17] S. Rehman, J. Comput. Appl. Math. 4, 446 (2014); ht tps://doi .org/10.4236/ajcm. 2014.45037.

[18] K. R. Grazier, W. I. Newman, F. Varadi, W. M. Kaula, J. M. Hyman, Icarus 140, 353 (1999); https://doi.or g/10.1006/icar.1999.6146.

[19] D. R. Kirsh, M. Duncan, R. Brasser, H. F. Levison, Icarus 199, 197 (2009); https://doi.org/10.1016/j. icarus.2008.05.028.

[20] M. S. Tiscareno, R. Malhotra, Astron. J. 138, 827 (2009); https://doi.org/10.1088/0004-6256/138/3/ 827.

[21] P. S. Lykawka, J. Horner, B. W. Jones, T. Mukai, Month. Not. R. Ast. Soc. 4, 1715 (2009); https://doi.org/10 $.1111 / \mathrm{j} .1365-2966.2009 .15243 . x$.

[22] D. A. Minton, R. Malhotra, Icarus 207, 744 (2010); ht tps : //doi .org/10.1016/j.icarus. 2009.12.008.

[23] P. W. Sharp, ACM Trans. Math. Softw. 32, 375 (2006); https://doi.org/10.1145/1163641.1163642.

\title{
НЕПЕРЕРВНІ НАБЛИЖЕННЯ ДЛЯ ДОВГОСТРОКОВИХ ЧИСЛОВИХ СИМУЛЯЦІЙ СОНЯЧНОї СИСТЕМИ
}

\author{
Ш. Рехман \\ Кафедра математики Інженерно-технологічного університету, Лахор, Пакистан
}

писано та проаналізовано ефективність різних комбінацій чотирьох числових інтеграторів вищого порядку та до дев'яти схем інтерполяції, застосованих до задачі, яка включає Сонце й чотири газові гіґанти (зовнішні планети), а саме: Юпітер, Сатурн, Уран та Нептун. Розглянуто схеми інтерполяції Ерміта з одним, двома та трьома часовими кроками й інтерполянтами для інтеграторів ODEX2 та ERKN. Інтерполянти є особливим прикладом схеми інтерполяції, що дають наближення, неперервне протягом одного кроку й на всьому інтервалі інтегрування. Інтерполянти досить вартісні порівняно з іншими схемами інтерполяції. Тому однією з цілей цієї праці є дослідження можливостей заміни інтерполянтів певних інтеграторів іншими схемами інтерполяції, можливо, ціною незначної втрати точності. Експерименти проведено, щоб дослідити зростання похибок у положеннях, швидкостях і відносній похибці енергії та кутового моменту з використанням різних комбінацій інтеграторів і схем інтерполяції на великому інтервалі інтегрування, аж до 100 мільйонів років для задачі Юпітера з локальним допуском помилок від $10^{-16}$ до $10^{-08}$.

Ключові слова: проблема Юпітера, схеми інтерполяції, довгострокове моделювання. 